\title{
BMJ Open Adapting to, integrating and self- managing HIV as a chronic illness: a scoping review protocol
}

\author{
Neo Phyllis Sematlane (D) , ${ }^{1}$ Lucia Knight, ${ }^{1,2}$ Caroline Masquillier, ${ }^{3}$ \\ Edwin Wouters (1) ${ }^{3,4}$
}

To cite: Sematlane NP, Knight L, Masquillier C, et al. Adapting to, integrating and self-managing HIV as a chronic illness: a scoping review protocol. BMJ Open 2021;11:e047870. doi:10.1136/ bmjopen-2020-047870

- Prepublication history and additional online supplemental material for this paper are available online. To view these files, please visit the journal online. To view these files, please visit the journal online (http://dx.doi.org/10.1136/ bmjopen-2020-047870).

Received 10 December 2020 Accepted 27 May 2021

Check for updates

(C) Author(s) (or their employer(s)) 2021. Re-use permitted under CC BY-NC. No commercial re-use. See rights and permissions. Published by BMJ.

${ }^{1}$ School of Public Health, University of the Western Cape Faculty of Community and Health Sciences, Bellville, South Africa

${ }^{2}$ Division of Social and Bahavioural Sciences, School of Public Health and Family Medicine, University of Cape Town, Rondebosch, South Africa ${ }^{3}$ Centre for Population, Family \& Health, Department of Sociology, Faculty of Social Sciences, University of Antwerp, Antwerp, Belgium

${ }^{4}$ Centre for Health Systems Research \& Development, University of the Free State, Bloemfontein, South Africa

Correspondence to Ms Neo Phyllis Sematlane; 3879990@myuwc.ac.za

\section{ABSTRACT}

Introduction The process of adapting to a life with a chronic illness, is a well-researched phenomenon for a number of common chronic illnesses. The construct, adaptation, embeds the notions of integration of the chronic illness into identity and self-management. Integration precedes self-management and is key to living positively with a chronic illness. Adaptation is an important concept in understanding trajectory and outcomes of living with a chronic illness. Applicability of these concepts to HIV as a chronic illness; when suppressive adherence has been achieved, however, is unknown. Specifically, the adaptation process to living with HIV as a chronic illness, the integration of HIV into identity and the resulting selfmanagement behaviours by adults living with HIV are relatively unexplored. We describe a protocol for a scoping review of adaptation to living with HIV, we structure the enquiry around integration of HIV into identity and self-management and interrogate theories, models and frameworks that have been proposed and studied and we evaluate them for relevance and usefulness in the care and management of HIV.

Methods and analysis Methods proposed by the Johanna Briggs Institute will be followed. The protocol was drafted using the Preferred Reporting Items for Systematic Reviews and Meta-analysis extension for Scoping Reviews and was registered with the Open Science Framework. MEDLINE, SCOPUS, Cochrane Library, CINHAL and SocINDEX databases will be searched. A search in Social Science Research Network eLibrary and Open Access Theses and Dissertations will gather grey literature and reference lists of included sources will be screened. Study selection process will involve a title and abstract review and full text review, guided by clearly defined inclusion and exclusion criteria.

Ethics and dissemination Ethical approval is not required because this is a proposed review and collection of data on publicly available materials. The results will be published in a topic relevant journal and presented at related scientific events.

\section{INTRODUCTION}

The efficacy of antiretroviral therapies (ART) in reducing HIV-related mortality and morbidity and ubiquitous availability of and access to ART treatments both in income rich and low-income and middle-income

\section{Strengths and limitations of this study}

This is an original scoping review protocol of concepts related to and assisting with understanding adaptation to living with HIV as a chronic illness by adult individuals living with the illness.

- The proposed review will explore literature on constructs of living with HIV as a chronic illness but also consider integration of HIV into identity and selfmanagement. It also attempts to map and document what may be beneficial to achieve sustained outcomes in HIV chronic illness care and management.

- Material identification and synthesis will include specialised databases and grey literature data, thus improving the comprehensiveness of the search.

- Exclusion of studies with certain key populations from the scope is necessary as these groups' characteristics may be potentially confounding factors in the adaptation process. This and the exclusion of studies documented in languages other than English may limit the review's richness.

countries (LMICs) effected a fundamental shift in the clinical management of HIV. A once fatal acute illness transformed into a manageable chronic illness and people living with HIV (PLHIV) on long-term therapy can reach a life expectancy comparable with that in the general population. ${ }^{12}$

To consider HIV as a chronic illness and to realise the best possible clinical outcomes, PLHIV on ART must achieve near perfect, lifelong adherence rates of $95 \%$ or more. ${ }^{34}$ Although challenges and barriers to good ART adherence have been elaborated on ${ }^{56}$ and effective strategies aimed at improving self-management for PLHIV have been identified, ${ }^{7}$ poor adherence to ART persists and is recognised as of critical global public health concern. ${ }^{8-10}$ Studies in all settings have reported ART adherence rates below the $95 \%$ rate required for optimal viral suppression. ${ }^{11} 12$ Additional evidence also suggests that adherence rates decline the longer PLHIV have been on treatment. ${ }^{911}$ 
Adherence to treatment and clinical outcomes are typically regarded as the most important indicators for evaluating the success or failure in the management of many chronic illnesses. ${ }^{13} 14$ HIV is no exception. The importance of adherence to ART and viral suppression are often first pieces of information or education patients are provided with once diagnosed. ${ }^{15}$ While this knowledge is crucial, this framing of adherence to treatment becomes problematic because it assumes the individual to be submissive and non-questioning subjects in their own care. ${ }^{1617}$ This biomedical approach to chronic illness management and adherence, can undermine the individual's personal experience, psychosocial evolution and growth related to living with a chronic illness. ${ }^{13} 16$

Learning to live with a chronic illness, referred to in this paper as adaptation, has been widely explored in previous research and there is extensive literature about the theory of living with a chronic illness. ${ }^{18-22}$ This concept is embedded in the definition of chronic illness itself, defined as the lived experience of long-term ill health. ${ }^{23}$ Chronic illnesses may be non-communicable (eg, diabetes) or communicable, as is the case for HIV, and encompass how people live and cope with the interference they bring. ${ }^{23}{ }^{24}$ The adaptation process is conceptualised in a range of different ways within the literature; biographical work, legitimation, adjustment and transitioning, among others. ${ }^{25-29}$ Despite descriptive differences, these conceptualisations commonly argue that the chronically ill individual must appraise and acknowledge their changed situation as a result of the illness, incorporate or integrate the chronic illness into their identity, lives and lifestyles and take action to change their personal circumstance. ${ }^{212528}$ Fundamentally, the ideal outcome of adaptation is for individuals to take control of their health and therefore live as well or positively as far as their chronic illness and context allow. ${ }^{30}{ }^{31}$ Pertinent to this review are the notions of and the inextricable link between integration of the chronic illness into identity and self-management or taking action to manage the illness by chronically ill individuals.

A number of studies have theorised about, reviewed and empirically tested the concept of integration of chronic illness into identity. ${ }^{17}{ }^{32-36}$ Integration of chronic illness into identity is defined as an ongoing process of reconciling the duality of self; merging the past non-ill self with the present chronically ill self and creating a new identity that is positively adapted to living with chronic illness. ${ }^{33} 37-39$ Integration has been shown to be the very premise of healing and ultimately living well with a chronic illness. ${ }^{34} 4041$ Moreover, it is because of integration of chronic illness into identity that chronically ill individuals are able to cultivate novel and constructive health patterns, successfully engage in health promoting behaviours and actively assume responsibility for their illness self-management. ${ }^{28} 39-42$

Self-management on the other hand is embedded in and could be perceived as both a characteristic of and an outcome of the process of integrating chronic illness into identity. ${ }^{21} 282943$ Studies have demonstrated selfmanagement as a descendent of integration, that integration precedes self-management and that without integration, the self-management processes and or outcomes may be suboptimal or not completed. ${ }^{3235}$ Others have shown how the undertaking of self-management related tasks of a chronic illness could be used to manage chronic illness identity issues. ${ }^{28}{ }^{44}$ Self-management theory considers the chronically ill individuals and their families as critical role players in their own care and treatment. ${ }^{45}$ With self-management, the assimilation of healthful behaviours into daily function and their continuous and consistent performance throughout the illness trajectory are deliberate, this with the aim of preventing or mitigating the potential negative impact of chronic illness $^{2845-48}$

Be that as it may, research into the broader construct of adaptation and the concepts of integration of chronic illness into identity and self-management, has tended to focus on common chronic illnesses such as rheumatoid arthritis ${ }^{2849}$ and diabetes. ${ }^{173450}$ With ubiquitous availability of and access to ART rendering HIV, a once fatal infectious illness, to become a manageable chronic illness ${ }^{1251}$ research interest has also progressed, although gradually, towards highlighting the importance of recognising the critical role of integration of HIV as a chronic illness into identity in its self-management. ${ }^{36-52}$ For instance, Wouters ${ }^{36}$ elevates the notion of integration (of HIV) into identity and proposes a theory of hybrid identity, that is, incorporation of HIV and ART into one hybrid identity that is susceptible to, yet resilient against changes caused by external and internal conditions. The hybrid identity may be used by PLHIV as a resilience resource and may in fact, potentially contribute towards sustained adherence to ART. ${ }^{36}$ On the other hand, Ho and $\mathrm{Goh}^{53}$ highlight gaps in the available literature, pointing to issues related to conceptual clarity, that the studies are not sufficiently underpinned by theory and that instances where there is rejection of an HIV identity should also be appreciated in the literature. ${ }^{53}$

However, the extent of applicability of the existing theoretical work on chronic illness adaptation, to HIV, has not been comprehensively explored. There is therefore a need to identify and map the extent to which HIV-related adaptation research has been conducted. To this end, the proposed review will take stock of the existing studies and will contribute a review of the scope of research on adaptation, including the concepts of integration into identity and self-management, in relation to HIV. Our primary objective is to identify, synthesise and summarise empirical research relating to adaptation to living with HIV as a chronic illness. Within the identified HIV adaptation literature, we explore and distill concepts, frameworks or theories that have been used or proposed, deliberately scrutinising the literature for integrating HIV into identity. We further categorise key characteristics related to integrating HIV into identity, including self-management aspects. We do this to achieve two undertakings: we 
first intend commenting on applicability, relevance and usefulness of these concepts to HIV as a chronic illness and second, we aim to locate and clarify the concept of HIV illness identity.

Studies with HIV positive adults 18 years and older, addressing the concept of adaptation as described, will be included in the review. We will consider HIV research conducted between the years 2000 and 2020, the period within which access to ART became widespread and rendered HIV a manageable chronic illness, ${ }^{1}$ particularly for LMICs. Although contexts may vary, studies conducted in both HICs and LMICs will be reviewed. Only studies published in English will be considered for inclusion.

\section{METHODS AND ANALYSIS}

\section{Study design and protocol}

The aim of this study is to understand the extent of knowledge in the area of adaptation, integration and selfmanagement in relation to HIV. A scoping review is the most appropriate approach as it maps concepts underpinning a research area and the main sources and types of evidence available. ${ }^{55}$ This study protocol is informed by the scoping review methods proposed by the Johanna Briggs Institute (JBI). ${ }^{5657}$

The Preferred Reporting Items for Systematic reviews and Meta-Analyses extension for Scoping Reviews format and checklist was employed for the drafting of this scoping review protocol. ${ }^{55}$ This protocol title was registered with the Open Science Framework on 2 August 2020 with registration number osf.io/3zv4f. Any significant changes made to the protocol will be documented.

\section{Eligibility criteria}

The inclusion criteria are based on the Population, Concept and Context framework as proposed by the JBI for scoping reviews as a less restricted alternative to the Population, Intervention, Comparator, Outcome framework. ${ }^{5657}$

\section{Population/types of participants}

The current scoping review will include review and empirical studies with either qualitative or quantitative content relevant to adaptation to living with HIV as a chronic illness and with reference to HIV positive adults 18 years and older, male or female. Studies including infants, children, adolescents and key populations, such as gay men, other men who have sex with men, sex workers, transgender people, people who inject drugs and prisoners ${ }^{58}$ as participants and those that report gender as a nonbinary concept will be excluded. This exclusion is based on the understanding that these personal demographic, social or sexual behavioural characteristics may influence how living with HIV illness is or is not experienced, and that these characteristics may therefore constitute confounding population factors.

\section{Concept}

The core and primary concept of interest for the proposed scoping review is adaptation to living with HIV as a chronic illness. We understand adaptation to be a broad concept that encompasses the notions of integration and self-management. We, therefore, will not limit our search to the two concepts. In addition, given our interest in the impact of the process of adaptation on the patient, a critical concern for our scoping review is to consider only those studies that document the lived experience of HIV, as told by or from the perspective of the patient. To this end, studies detailing living with HIV from the family and or a professional's perspective will be excluded. The scoping review will additionally purposively seek and extract from those selected HIV adaptation studies, any emerging aspects of integration and patient selfmanagement, as a result of the adaptation process. Lack of reference to either integration or self-management will however not constitute an exclusion criterion.

\section{Context}

The primary focus of the scoping study is HIV as a chronic illness. To this end, adaptation studies of other chronic illness will not be included in the scoping review. In addition, searches for the review will be contained to studies conducted between the years 2000 and 2020; a period during which HIV had largely been classified as a manageable chronic illness simultaneously in both HICs and LMICs.

\section{Information sources and search strategy}

The literature will be sourced through searches of: (1) electronic bibliographic databases; (2) grey literature and (3) screening of reference lists of included documents. First, the databases, MEDLINE, SCOPUS and Cochrane Library, will be searched as they have been recommended to be the ideal database combination for expansive coverage. ${ }^{5960}$ Additionally, because the topic under review, adaptation to living with HIV as a chronic illness, straddles disciplines, we will also search the CINAHL and SocINDEX (via EbscoHost) databases. Second, a grey literature search of Social Science Research Network (SSRN) eLibrary and Open Access Theses and Dissertations will also be conducted. Finally, screening of reference lists of included documents for relevant articles will be undertaken. This comprehensive search approach was selected to exhaust all possible information on the subject. Types of published peer-review articles eligible for inclusion will include original research, review articles, short reports and case studies.

\section{Electronic bibliographic databases}

An experienced research librarian provided expert input into the development of the search strategy. An analysis of the Medical Subject Headings terms and the words in the title, abstract, and keyword sections of a subsample of relevant literature, about adaptation to living with HIV and or chronic illness, ${ }^{21} 25295461-63$ informed the search strategy design. In addition, the search will include all identified synonyms for adaptation, to optimise sensitivity. Using an iterative approach, the research team 
will review preliminary search results for relevance and will suggest modifications and improvements before the search strategy is finalised. The search strategy will be validated using the same subsample of papers. A sample search strategy developed for PubMed (detailed in online supplemental appendix I), will be refined for use in the other selected electronic databases, with assistance from the librarian. The lead reviewer will perform the final searches from inception to date of search, export the search results into Mendeley Desktop and remove all duplicates.

\section{Grey literature Search}

To preclude publication bias and to further explore the breadth of information on the topic, a grey literature search will also be conducted. A systematic search of the SSRN will collect any available preprints on the subject and via the Open Access Theses and dissertations platform, we will gather dissertations and theses with relevant content.

\section{Reference list screening}

Reference lists of all included sources will be screened for potential additional sources.

\section{Source selection}

For the purposes of this proposed scoping review, we have adopted and customised the JBI template for source of evidence details, characteristics and results extraction instrument (online supplemental appendix II). The adapted extraction tool will be used in both the initial stages of study screening (to confirm study relevance) and selection and the later phase of data extraction from the selected studies. To ensure systematic and reproducible study selection and data charting processes and to foster high inter-rater reliability, a calibration exercise will be undertaken. For this pilot, first the review lead will use a seminal article to ascertain if the extraction instrument is appropriate for its intended use. Once confidence with the tool has been internally established, all members of the review team be involved in the pilot of the extraction tool, using a minimum of twenty abstracts to review titles and abstracts against the above-mentioned inclusion criteria. We will review the results of the calibration, discuss any discrepancies among reviewers and make refinements to the extraction tool as identified and required. We will then share the balance of the search results among the reviewers in the team for duplicate independent screening. Reviewers will at the same time document reasons for exclusion on the extraction form and progress those articles considered relevant and eligible, to the second phase of full-text screening. Confirmed sources for inclusion in the scoping review will then be moved to the final stages of data extraction, charting and synthesis.

\section{Data extraction, charting and synthesis and presentation of results}

The customised data extraction tool (online supplemental appendix II) will be used to collect relevant information on: (1) key study characteristics (eg, publication year, publication type, study design, country, patient population characteristics); (2) detailed information on the definition and words and terms used to describe adaptation or living with HIV anywhere in the article and (3) information on words and terms used to describe any aspect of HIV self-management anywhere in the article. The lead reviewers have formulated a matrix of variables that will form the basis for data extraction (table 1).

Following identification and selection of relevant HIV adaptation literature, we will explore and isolate within those studies, the theories, constructs and models and frameworks that have been applied or proposed. Subsequently, we will scrutinise and extricate from those theories, constructs and models any aspects related and relevant to integration into identity and self-management of HIV. We aim to provide a descriptive summary of what has been foregrounded in the area of living with HIV as a chronic illness, and offer commentary on applicability, relevance and usefulness of discovered concepts within the literature. Where relevant, we intend suggesting how these concepts may be adapted specifically for HIV as a chronic illness.

The reviewers will independently chart data in duplicate from each eligible article. Should there be any disagreements among the reviewers, these will be resolved through discussion or in case of an impasse, the supervising researcher will provide final adjudication. Data charting will be implemented using Microsoft Excel. Throughout these processes of screening, data extraction and charting, the supervising researcher will provide oversight and perform frequent and random checks. A narrative description and summary of the search decision process, study identification and inclusion decision flow chart; aims of the reviewed sources, concepts adopted and results that relate to the review objective will precede the presentation of the results. Importantly, the relevance of isolated data, together with supporting evidence for the implications to the review objectives will be presented, for included sources. A final meeting will be held between the lead reviewer, senior supervising researcher and members of the review team to review study results, discuss any refinements proposed and finally approve the final outcome of the study.

\section{Risk of bias assessment or quality appraisal}

Consistent with the JBI scoping review methodology and as this is a scoping review that aims to map all available knowledge regarding adaptation and self-management in relation to HIV, we will not perform a risk of bias assessment. Although not routine for scoping reviews, we will however appraise the quality of the selected articles, reviews, reports and case studies. The JBI provides a number of critical appraisal tools, in the form of checklists, to evaluate the relevance, reliability and results of the selected published papers. A number of these checklists will be referred to and deployed in evaluating the quality of the potential studies for inclusion in the review. ${ }^{64}$ Documents retrieved through the grey literature searches will not be appraised. 
Table 1 Matrix of sample variables related to the antecedent concept (integration of HIV) and descendent concept (selfmanagement of HIV), adapted from Schulman-Green et $a l^{48}$ and organised according to the dimensions within the individual and family self-management theory ${ }^{45}$

\begin{tabular}{|c|c|c|c|}
\hline \multirow{2}{*}{$\begin{array}{l}\text { Adaptation/ } \\
\text { living with HIV } \\
\text { Self-mManagement }\end{array}$} & \multicolumn{3}{|c|}{$\begin{array}{l}\text { Adjustment, transitioning, acceptance, integration, incorporation, coping, making meaning, } \\
\text { enrichment/growing as a person as a result of HIV, Identity }\end{array}$} \\
\hline & $\begin{array}{l}\text { Context } \\
\text { dimension }\end{array}$ & $\begin{array}{l}\text { Presence of family, friends } \\
\text { healthcare providers and } \\
\text { community }\end{array}$ & $\begin{array}{l}\text { Social support (ability and capacity to enlist illness } \\
\text { related help, for example, family member assists with } \\
\text { collecting chronic medication from healthcare facility } \\
\text { on behalf of patient) } \\
\text { Communication, relationship and service provision } \\
\text { coordination } \\
\text { Spiritual support, capacity to address social and } \\
\text { environmental challenges (eg, stigma) }\end{array}$ \\
\hline & $\begin{array}{l}\text { Process } \\
\text { dimension }\end{array}$ & $\begin{array}{l}\text { Education about HIV and } \\
\text { taking Ownership }\end{array}$ & $\begin{array}{l}\text { Acquiring information about HIV } \\
\text { Learning about individual regimen } \\
\text { Learning about body's responses (side effects from } \\
\text { treatment) } \\
\text { Becoming an 'expert' about HIV }\end{array}$ \\
\hline
\end{tabular}

\section{Patient and public involvement}

Given the methodological focus of this study, patients or the public were not involved in the design or drafting of our protocol or conduct, or reporting, or dissemination plans of our research.

\section{DISCUSSION}

The review will allow for the identification, synthesis and summary of existing knowledge on adaptation to living with HIV as a chronic illness and specifically explore the concepts of integration and self-management for adult PLHIV. This review will enable the elaboration of a comprehensive repository of constructs used to describe the HIV chronic illness experience. In addition, because we also explore the usefulness and relevance of integration and self-management to HIV as a chronic illness, an adapted framework for managing HIV as a chronic illness, premised on these two concepts and informed by the scoping review will be developed.

\section{Application of results}

At a broader theoretical level, the review work will build on a growing body of literature that examines the concept of adaptation to living with a chronic illness, specifically in our case, what may be applicable to HIV as a chronic illness. A unique aspect of the current scoping review is that it not only explores literature on constructs of living with HIV as a chronic illness but by framing the scope against the concepts of integration and self-management, it also attempts to map and document what may be beneficial for sustained outcomes in HIV chronic illness management. Evaluating whether chronically ill individuals successfully adapt to living with chronic illness and their attempts at attaining the ideal of positive living in their illness trajectory, particularly for HIV, is an area within chronic illness care that is relatively unexplored. Exploring and expanding knowledge on these concepts therefore may strengthen and improve HIV chronic care and management.

At a practical level, this work may inform an approach to HIV chronic care by healthcare professionals that recognises the critical role of HIV illness adaptation and integration and its relation to self-management and eventual impact on patient outcomes. Furthermore, knowledge gathered from the study may contribute towards a framework that could guide healthcare professionals and significant others in facilitating and assisting PLHIV to successfully adapt to living with HIV, with the aim of assuring better outcomes for the patient.

\section{Potential implications and next steps}

This scoping review work will form part of and be the initial stage of a multiphase research study aimed at 
investigating the relationship between the construct of HIV illness identity; the extent to which the ill individual has integrated their HIV chronic illness diagnosis into their identity or sense of self, and patient outcomes such as sustained ART adherence. To this end, the scoping review will situate the HIV illness identity construct within the broader adaptation to living with a chronic illness body of literature.

\section{Ethics and dissemination}

This scoping review forms an initial stage of a multiphase doctoral research study and is also part of the Sinako cluster randomised control study, for which ethics approval has been obtained.$^{65}$ Results of the proposed review will be published in a peer-reviewed journal and presented at related scientific events.

Acknowledgements The authors would like to thank Ms Karen Cook who assisted with the search strategy.

Contributors NPS conceived and developed the study objectives. NPS, LK, CM and EW contributed meaningfully to the drafting and editing of the protocol, reviewed and revised the final version of the protocol. All authors, NPS, LK, CM and EW approved the final manuscript.

Funding The financial assistance of the VLIR-UOS and Belgian Development Cooperation (Grant Number FWO G035018N) towards this research is hereby acknowledged. Opinions expressed and conclusions arrived at, are those of the author and are not necessarily to be attributed to the SOPH, VLIR or DGD. The work reported herein was made possible through funding by the South African Medical Research Council (SAMRC) through its division of Research Capacity Development under the National Health Scholars Programme from funding received from the Public Health Enhancement Fund/ South African National Department of Health (SAMRC Project Code 57041).

Disclaimer The content hereof is the sole responsibility of the authors and does not necessarily represent the official views of the SAMRC.

Competing interests None declared.

Patient consent for publication Not required.

Provenance and peer review Not commissioned; externally peer reviewed.

Supplemental material This content has been supplied by the author(s). It has not been vetted by BMJ Publishing Group Limited (BMJ) and may not have been peer-reviewed. Any opinions or recommendations discussed are solely those of the author(s) and are not endorsed by BMJ. BMJ disclaims all liability and responsibility arising from any reliance placed on the content. Where the content includes any translated material, BMJ does not warrant the accuracy and reliability of the translations (including but not limited to local regulations, clinical guidelines, terminology, drug names and drug dosages), and is not responsible for any error and/or omissions arising from translation and adaptation or otherwise.

Open access This is an open access article distributed in accordance with the Creative Commons Attribution Non Commercial (CC BY-NC 4.0) license, which permits others to distribute, remix, adapt, build upon this work non-commercially, and license their derivative works on different terms, provided the original work is properly cited, appropriate credit is given, any changes made indicated, and the use is non-commercial. See: http://creativecommons.org/licenses/by-nc/4.0/.

\section{ORCID iDs}

Neo Phyllis Sematlane http://orcid.org/0000-0001-8511-619X

Edwin Wouters http://orcid.org/0000-0003-2268-3829

\section{REFERENCES}

1 Deeks SG, Lewin SR, Havlir DV. The end of AIDS: HIV infection as a chronic disease. Lancet 2013;382:1525-33.

2 Mahungu TW, Rodger AJ, Johnson MA. HIV as a chronic disease. Clin Med 2009;9:125-8.
3 Simoni JM, Amico KR, Pearson CR, et al. Strategies for promoting adherence to antiretroviral therapy: a review of the literature. Curr Infect Dis Rep 2008;10:515-21.

4 Paterson DLet al. Adherence to anti-HIV therapy and the outcome of treatment. Ann Intern Med 2013;133:21.

5 Reda AA, Biadgilign S. Determinants of adherence to antiretroviral therapy among HIV-infected patients in Africa. AIDS Res Treat 2012;2012:1-8.

6 Wekesa E. Art adherence in resource poor settings in sub-Saharan Africa: a multi- disciplinary review, 2007. Available: http://uaps2007. princeton.edu/papers/70123.

7 Areri HA, Marshall A, Harvey G. Interventions to improve selfmanagement of adults living with HIV on antiretroviral therapy: a systematic review. PLoS One 2020;15:e0232709.

8 World Health Organization, Sabaté E. Adherence to long-term therapies : evidence for action. Geneva: World Health Organization, 2003.

9 Bartlett JA. Addressing the challenges of adherence. J Acquir Immune Defic Syndr 2002;29 Suppl 1:S2-10.

10 Eshun-Wilson I, Rohwer A, Hendricks L, et al. Being HIV positive and staying on antiretroviral therapy in Africa: a qualitative systematic review and theoretical model. PLoS One 2019;14:e0210408.

11 O'Connor JL, Gardner EM, Mannheimer SB, et al. Factors associated with adherence amongst 5295 people receiving antiretroviral therapy as part of an international trial. $J$ Infect Dis 2013:208:40-9.

12 Mills EJ, Nachega JB, Buchan I, et al. Adherence to antiretroviral therapy in sub-Saharan Africa and North America. JAMA 2006;296:679-90.

13 Leventhal H, Halm E, Horowitz C. Living with chronic illness: a contextualized, self-regulation approach. In: Sutton AB, Johnston M, eds. The SAGE Handbook of health psychology. Thousand Oaks: Sage, 2015.

14 Engel G. The need for a new medical model: a challenge for biomedicine. Science 1977;196:129-36.

15 Perazzo J, Reyes D, Webel A. A systematic review of health literacy interventions for people living with HIV. AIDS Behav 2017;21:812-21.

16 Hernandez CA. The experience of living with insulin-dependent diabetes: lessons for the diabetes educator. Diabetes Educ 1995;21:33-7.

17 Whittemore R, Sister Callista Roy. Adapting to diabetes mellitus: a theory synthesis. Nurs Sci Q 2002;15:311-7.

18 Bury M. Chronic illness as biographical disruption. Sociol Health IIIn 1982;4:167-82.

19 Charmaz K. Self in chronic illness. Occup Ther J Res 2002;22:31-41.

20 Corbin JM. Accompaniments of chronic illness: changes in body, self, biography, and biographical time. Res Sociol Health Care 1987;6:249-81.

21 Ambrosio L, Senosiain García JM, Riverol Fernández M, et al. Living with chronic illness in adults: a concept analysis. J Clin Nurs 2015;24:2357-67.

22 Pierret J. The illness experience: state of knowledge and perspectives for research. Sociol Health IIIn 2003;25:4-22.

23 Martin CM. Chronic disease and illness care. Can Fam Med 2007;53:2086-91.

24 Walker $\mathrm{C}$. Recognising the changing boundaries of illness in defining terms of chronic illness: a prelude to understanding the changing needs of people with chronic illness. Aust Health Rev 2001;24:207-14.

25 Bury M. The sociology of chronic illness: a review of research and prospects. Sociol Health \& IIIness 1991;13:451-68.

26 Sharpe L, Curran L. Understanding the process of adjustment to illness. Soc Sci Med 2006;62:1153-66.

27 Kralik D, Visentin K, van Loon A. Transition: a literature review. J Adv Nurs 2006;55:320-9.

28 Kralik D, Koch T, Price K, et al. Chronic illness self-management: taking action to create order. J Clin Nurs 2004;13:259-67.

29 Sidell NL. Adult adjustment to chronic illness: a review of the literature. Health Soc Work 1997;22:5-11.

30 Livneh H. Psychosocial adaptation to chronic illness and disability: a conceptual framework. Rehabilitation Counseling Bulletin 2001;44:151-60.

31 Weinert C, Cudney S, Spring A. Evolution of a conceptual model for adaptation to chronic illness. J Nurs Scholarsh 2008;40:364-72.

32 Adams S, Pill O, Jones A. Medication, chronic illness and identity: the perspective O, F people with asthma. Science 1997;45:189-201.

33 Goldman JB, Maclean HM. The significance of identity in the adjustment to diabetes among insulin users. Diabetes Educ 1998;24:741-8.

34 Hernandez CA. Integration: the experience of living with insulin dependent (type 1) diabetes mellitus. Canadian Journal of Nursing Research Archive 2000;28:220-8. 
35 Tilden B, Charman D, Sharples J, et al. Identity and adherence in a diabetes patient: transformations in psychotherapy. Qual Health Res 2005;15:312-24.

36 Wouters E. Life with HIV as a chronic illness: a theoretical and methodological framework for antiretroviral treatment studies in resource-limited settings. Soc Theory Health 2012;10:368-91.

37 Kralik D. The quest for ordinariness: transition experienced by midlife women living with chronic illness. J Adv Nurs 2002;39:146-54.

38 Westra BL, Rodgers BL. The concept of integration: a foundation for evaluating outcomes of nursing care. J Prof Nurs 1991;7:277-82.

39 Whittemore R, Dixon J. Chronic illness: the process of integration. $J$ Clin Nurs 2008;17:177-87.

40 Medich CJ, Stuart E, Chase SK. Healing through integration: promoting wellness in cardiac rehabilitation. J Cardiovasc Nurs 1997:11:66-79.

41 Aujoulat I, Marcolongo R, Bonadiman L, et al. Reconsidering patient empowerment in chronic illness: a critique of models of self-efficacy and bodily control. Soc Sci Med 2008;66:1228-39.

42 Fleury JD. Empowering potential: a theory of wellness motivation. Nurs Res 1991;40:286-91.

43 Samson A, Siam H. Adapting to major chronic illness: a proposal for a comprehensive task-model approach. Patient Educ Couns 2008;70:426-9.

44 Maietta JT. Integrating illness management into identity verification processes. Qual Health Res 2021;31:254-70.

45 Ryan P, Sawin KJ. The individual and family self-management theory: background and perspectives on context, process, and outcomes. Nurs Outlook 2009;57:217-25.

46 Grady PA, Gough LL. Self-management: a comprehensive approach to management of chronic conditions. Am J Public Health 2014:104:e25-31.

47 Lorig KR, Holman H. Self-management education: history, definition, outcomes, and mechanisms. Ann Behav Med 2003;26:1-7.

48 Schulman-Green D, Jaser S, Martin F, et al. Processes of selfmanagement in chronic illness. J Nurs Scholarsh 2012;44:136-44.

$49 \mathrm{Li} \mathrm{J}$, Drury V, Taylor B. A systematic review of the experience of older women living and coping with type 2 diabetes. Int J Nurs Pract 2014;20:126-34.

50 Shaul MP. From early twinges to mastery: the process of adjustment in living with rheumatoid arthritis. Arthritis Care Res 1995;8:290-7.

51 World Health Organization. HIV: from a devastating epidemic to a manageable chronic disease. in ten years in public health, 2007-
2017: report by DR Margaret Chan, Director-General, World Health Organization. Geneva: World Health Organization, 2017.

52 Baumgartner LM. The incorporation of the HIV/AIDS identity into the self over time. Qual Health Res 2007;17:919-31.

53 Ho LP, Goh EC. Using identity theory to examine the function of normative identities in living with HIV. Heal Soc Work 2018;43:274-7.

54 Laws MB. Explanatory models and illness experience of people living with HIV. AIDS Behav 2016;20:2119-29.

55 Tricco AC, Lillie E, Zarin W, et al. PRISMA extension for scoping reviews (PRISMA-ScR): checklist and explanation. Ann Intern Med 2018;169:467-73.

56 Peters MDJ, Godfrey CM, Khalil H, et al. Guidance for conducting systematic scoping reviews. Int J Evid Based Healthc 2015;13:141-6.

57 Peters M, Godfrey CM, Mcinerney P, et al. 2017 guidance for the conduct of JBI scoping reviews. Joana Briggs Inst Rev 2017;13:141-6.

58 UNAIDS. UNAIDS 2015 terminology guidelineshttps://www.unaids. org/sites/default/files/media_asset/2015_terminology_guidelines_en. pdf

59 Institute of Medicine,. Finding what works in health care: standards for systematic reviews. Washington, DC: The National Academies Press, 2011.

60 Bayliss SE, Davenport CF, Pennant ME. Where and how to search for information on the effectiveness of public health interventions--a case study for prevention of cardiovascular disease. Health Info Libr J 2014;31:303-13.

61 Bova C. Adjustment to chronic illness among HIV-infected women. J Nurs Scholarsh 2001;33:pp.:217-24.

62 Siegel K, Lekas H-M. Aids as a chronic illness: psychosocial implications. AIDS 2002;16 Suppl 4:S69-76.

63 Wouters E, De Wet K. Women's experience of HIV as a chronic illness in South Africa: hard-earned lives, biographical disruption and moral career. Sociol Health IIIn 2016;38:521-42.

64 The Joanna Briggs Institute. Checklist for qualitative research. Adelaide, Australia: The Joanna Briggs Institute, 2017. https://jbi. global/sites/default/files/2019-05/JBI_Critical_Appraisal-Checklist_ for_Qualitative_Research2017_0.pdf

65 Masquillier C, Knight L, Campbell L, et al. Sinako, a study on HIV competent households in South Africa: a cluster-randomised controlled trial protocol. Trials 2020;21:154. 